\section{Hut ab vor dem politischen Engagement der Zürcher Ärzte!}

Während Monaten wurde im Kanton Zürich eine heftige politische Auseinandersetzung zur Frage geführt, wer den Patientinnen und Patienten Medikamente abgeben darf. Zur Diskussion stand eine im Kantonsrat beschlossene Regelung, bei der Ärzte nur noch dann berechtigt gewesen wären, wenn sie am allgemein-medizinischen Notfalldienst teilgenommen und zusätzlich mehr als 500 Meter von der nächsten Apotheke entfernt praktiziert hätten. Gemäss einer geographischen Auswertung wären über 2000 Zürcher Ärzte zukünftig gezwungen gewesen, ihren Patientinnen und Patienten Rezepte auszustellen. Nur noch ganz wenige Ärzte hätten in der Sprechstunde, bei Hausbesuchen und im Notfalldienst das Recht zur Medikamentenabgabe gehabt. Solch einschränkende, willkürliche und bürokratische Kriterien galt es zu bekämpfen. Die Zürcher Ärzteschaft führte deshalb gemeinsam mit Politikerinnen und Politikern einen engagierten Abstimmungskampf. Mit Erfolg! Die Stimmbürgerinnen und Stimmbürger lehnten die von Parlament und Regierung gutgeheissene und von den meisten Zürcher Medien unterstützte Vorlage ab.

Dieser Erfolg war nur möglich, weil die Ärzteschaft sich geschlossen, engagiert und zielgerichtet gegen dieses Vorhaben eingesetzt hatte. Getreu dem Motto "Politik lässt sich nicht delegieren" beteiligten sich zahlreiche Ärzte aktiv am Abstimmungskampf. In den Praxen, an Standaktionen, an Veranstaltungen und in den Medien wurden die negativen Konsequenzen dieses Erlasses erläutert. Ein gleiches geschah bei den Parolenfassungen der Parteien. Auch dort traten einzelne Ärzte erfolgreich als Podiumsteilnehmer oder Votanten auf. Die bürgerlichen Parteien empfahlen schliesslich ein "Nein", während die linken Parteien "Stimmfreigabe" gewährten.

Eine erste Analyse der Abstimmung vom 23. September zeigt, dass die ärztliche Medikamentenabgabe sehr geschätzt wird. Dies insbesondere aufdem Land. Je nach Gemeinde und Engagement der Ärzte konnten dort Nein-Stimmenanteile von bis zu $80 \%$ erreicht werden. Aber auch in den Städten Zürich und Winterthur, in denen die ärztliche Medikamentenabgabe derzeit noch verboten und somit unbekannt ist, wurden respektable Ergebnisse erzielt.

Nach dieser erfolgreichen Abstimmung käme ein neuerlicher Versuch zur Einschränkung der ärztlichen Medikamentenabgabe einer Missachtung des Volkswillens gleich. Denn das Zürcher Stimmvolk hat jetzt entschieden! Kranke Menschen sollen - insbesondere auf dem Land - weiterhin die freie Wahl haben, wo sie ihre Medikamente beziehen möchten. Dieses erfreuliche Ergebnis ist nicht zuletzt dem geschlossenen und engagierten Einsatz der Zürcher Ärzte zu verdanken. Sie haben eindrücklich und exemplarisch gezeigt, wie politische Ziele erreicht werden können. Gratulation!

Dr. Sven Bradke, Ärzte mit Patientenapotheke (APA) 